\title{
Neoadjuvant chemotherapy reduces blood loss during the resection of pediatric choroid plexus carcinomas
}

\author{
${ }^{*}$ Christian Schneider, MD, ${ }^{1}$ lan Kamaly-Asl, MD, MBChB, FRCS(SN), ${ }^{2}$ Vijay Ramaswamy, MD, ${ }^{3}$ \\ Lucie Lafay-Cousin, MD, MSc, ${ }^{4}$ Abhaya V. Kulkarni, MD, PhD, ${ }^{1}$ James T. Rutka, MD, PhD, ${ }^{1,3}$ \\ Marc Remke, MD, ${ }^{3}$ Daniel Coluccia, MD, ${ }^{3}$ Uri Tabori, MD, ${ }^{3,5}$ Cynthia Hawkins, MD, PhD, ${ }^{3,6}$ \\ Eric Bouffet, MD, ${ }^{3}$ and Michael D. Taylor, MD, PhD ${ }^{1,3}$
}

\begin{abstract}
Divisions of ${ }^{1}$ Neurosurgery, ${ }^{5}$ Neurooncology, and ${ }^{6}$ Neuropathology, ${ }^{3}$ Arthur and Sonia Labatt Brain Tumor Research Center, The Hospital for Sick Children, Toronto, Ontario; ' ${ }^{4}$ Department of Pediatric Oncology and Bone Marrow Transplantation, Alberta Children's Hospital, Calgary, Alberta, Canada; and 'Division of Neurosurgery, Royal Manchester Children's Hospital, Manchester, England
\end{abstract}

OBJECT Choroid plexus carcinomas (CPCs) are rare brain tumors originating from the ventricular choroid plexus. They account for $2 \%-4 \%$ of all pediatric brain tumors and are most frequently seen in very young children. This pediatric proclivity, in combination with a marked vascularity, renders an aggressive resection a difficult and often dangerous endeavor. Blood losses of several total blood volumes in small children are not uncommon, sometimes forcing the neurosurgeon to abort the procedure, often leaving residual tumor. Great extent of tumor resection is an accepted beneficial factor for overall survival. Therefore, a second resection usually follows the administration of adjuvant chemotherapy. Second-look surgery appears to be associated with markedly decreased blood loss. Histological examination of specimens obtained at a second intervention shows decreased vascularity and fibrotic changes in tumor tissue. At the Hospital for Sick Children in Toronto, this empirical finding led to the strategy of neoadjuvant chemotherapy to minimize blood loss and maximize cytoreduction. The authors undertook this study to assess the potentially beneficial effect of neoadjuvant chemotherapy on blood loss during surgery for CPCs.

METHODS In this retrospective cohort review, the demographic, clinical, and treatment parameters of 22 consecutive patients diagnosed with CPC are presented. All underwent surgical treatment at the Hospital for Sick Children from 1982 to 2013. Special attention was given to the impact of neoadjuvant chemotherapy on extent of resection and intraoperative blood loss. Extent of resection was calculated based on perioperative neuroimaging, and amount of blood loss was estimated based on transfusion parameters and perioperative changes in hematocrit.

RESULTS Ten patients did not receive neoadjuvant chemotherapy, and 12 were treated with 2-5 cycles of ICE (ifosfamide, carboplatin, etoposide) chemotherapy in a neoadjuvant fashion. The 22 patients included in the study underwent a total of 37 tumor resection surgeries. In all of the cases in which neoadjuvant chemotherapy was used, at least a near-total resection (> 95\% of tumor volume) was achieved. Patients who underwent gross-total resection had prolonged overall survival. Of the 37 resections, 18 were performed after chemotherapy. Mean blood loss in the neoadjuvant chemotherapy group was $22 \%$ of total estimated blood volume as opposed to $96 \%$ in patients without preoperative chemotherapy.

CONCLUSIONS In children with CPC, the administration of neoadjuvant chemotherapy decreases intraoperative blood loss and increases extent of resection with a significant positive effect on overall survival.

http://thejns.org/doi/abs/10.3171/2014.12.PEDS14372

KEY WORDS choroid plexus carcinoma; neoadjuvant chemotherapy; blood loss; children; surgical treatment; oncology

ABBREVIATIONS chemo = chemotherapy; CPC = choroid plexus carcinoma; EOR = extent of resection; ICE = ifosfamide, carboplatin, and etoposide. SUBMITTED July 19, 2014. ACCEPTED December 30, 2014.

INCLUDE WHEN CITING Published online May 1, 2015; DOI: 10.3171/2014.12.PEDS14372.

DISCLOSURE The fellowship and research projects of Christian Schneider are supported by the Novartis Foundation for Medical-Biological Research, Basel, Switzerland. Dr. Taylor is supported by funds from the Garron Family Chair in Childhood Cancer Research at the Hospital for Sick Children and the University of Toronto and by operating funds from the National Institutes of Health (R01CA159859 and R01CA148699), the Terry Fox Research Institute, the Canadian Institutes of Health Research, and the Pediatric Brain Tumor Foundation.

* Drs. Schneider and Kamaly-Asl contributed equally to this work. 
$\mathrm{C}$ HOROID plexus carcinomas (CPCs) are rare malignant brain tumors originating from the ventricular choroid plexus. They account for $2 \%-4 \%$ of all childhood brain tumors and have an increased incidence in very young children and infants. ${ }^{4,9,12,17}$ The treatment of these WHO Grade III neoplasms is difficult for various reasons. First, they show an exquisite vascularity, which-in combination with the tumor's predilection for infants - renders the attempt at an aggressive resection difficult and often dangerous..$^{9,15-17}$ Second, the young age of the patients usually prohibits radiotherapy, an otherwise rather effective treatment for these tumors., ${ }^{1,6,17,18}$ Finally, the involvement of the choroid plexus interferes with the cerebrospinal fluid (CSF) circulation, often leading to decompensated hydrocephalus at presentation, requiring emergent surgical treatment under nonoptimal circumstances. ${ }^{2}$

The achievement of a gross-total resection in CPC is widely accepted to be beneficial in terms of overall and possibly also progression-free survival. ${ }^{16,17}$ The role of adjuvant chemotherapy and radiotherapy is still a matter of debate. The low incidence of CPC impedes large-scale studies, and so far, no standard adjuvant treatment protocol exists. ${ }^{7,11,17}$ At the Hospital for Sick Children in Toronto, the ICE regimen (ifosfamide, carboplatin, and etoposide) has been administered as first-line adjuvant chemotherapy for 3 decades. ${ }^{10}$ The primary intention was to treat residual tumor in patients in whom a gross-total resection could not be achieved or to treat disseminated disease. However, when a second attempt at resection was made after the administration of adjuvant chemotherapy, it appeared that tumor vascularity was significantly reduced. Grosstotal resection seemed to be easier and blood loss less of a concern. ${ }^{15}$ Histological assessment of the tumor tissue from these second resections showed fibrotic changes and a markedly decreased vascularity. ${ }^{10}$ This empirical finding-also reported in the literature-eventually led to an institutional change in management, with the administration of ICE chemotherapy in a neoadjuvant fashion. ${ }^{10,13,14}$ In 1999, the first patient underwent an intentional diagnostic biopsy with tumor resection delayed until several cycles of neoadjuvant ICE chemotherapy were administered. Whenever clinically possible, this approach has been pursued since then. The rate of gross-total resections seemed to improve - as reported by Lafay-Cousin et al.--based on the patients treated at the Hospital for Sick Children until $2006 .^{10}$ The aim of this retrospective review is to update those data with information on the patients treated at our institution to date. In addition, special attention will be directed to the impact of neoadjuvant chemotherapy on intraoperative blood loss and overall survival..$^{10,16,17}$

\section{Methods Study Design}

Given the low incidence of CPC, a retrospective cohort review design had to be chosen. The Department of Neurooncology database was searched for the histological diagnosis of CPC. After permission from the institutional research ethics board was obtained, a chart review was performed for demographic, clinical, and treatment parameters. In addition, tumor size and extent of resection were calculated from the patients' perioperative neuroimaging studies. Microsoft Access 2013 was used for data entry and organization.

For the assessment of the impact of neoadjuvant chemotherapy, group comparison was performed in 2 different ways. First, all patients were stratified into 2 groups, one with and one without neoadjuvant chemotherapy ("patients with neoadjuvant chemo" and "patients without neoadjuvant chemo"). The neoadjuvant chemo group was defined as including all patients who underwent at least 1 additional resection within 1 year of chemotherapy (i.e., within the first 12 months after completion of the course of treatment), regardless of the type of their first surgical intervention.

The second comparison addressed surgical parameters to assess blood loss in the actual surgical procedures. Similar to the group comparison described above, all resections were stratified into two groups based on the use of neoadjuvant chemotherapy, although this time the analysis was by procedure (i.e., "resection following neoadjuvant chemo" and "resection without neoadjuvant chemo"). Again, resection had to follow chemotherapy within 1 year.

\section{Patients With and Without Neoadjuvant Chemotherapy}

In addition to demographic parameters, extent of tumor resection (EOR) and patient survival data were analyzed. To assess the impact of EOR on survival, measurements of pre- and postoperative tumor volumes were performed. Tumor volumes were approximated with the following formula: $\mathrm{V}=1 / 6 \times \pi \times \mathrm{D} 1 \times \mathrm{D} 2 \times \mathrm{D} 3$, where $\mathrm{V}$ represents volume and D1, D2, and D3 represent the largest diameter in all 3 dimensions measured on contrast-enhanced MRI or CT imaging. For every surgical intervention except diagnostic biopsies, the percentage of resected tumor volume was calculated. Gross-total resection was defined as no visible contrast-enhancing lesion on neuroimaging according to the neuroradiologist's assessment. Near-total resection implied a residual tumor volume of less than $5 \%$, and partial resection a residual tumor volume of $5 \%$ or more.

In patients who underwent several surgeries, the EOR data for the first tumor resection surgery was used in the "patients without neoadjuvant chemo" group, and data for the first resection after chemotherapy in the "patients with neoadjuvant chemo" group. Kaplan-Meier survival curves were then calculated with the parameters neoadjuvant chemotherapy and EOR as factors for comparison.

\section{Resections With and Without Neoadjuvant Chemotherapy}

To assess the effect of neoadjuvant chemotherapy on individual surgical procedures in terms of blood loss and EOR, every resection was then evaluated separately. Similar to the definition above, resections were stratified into those in which resection followed chemotherapy within 1 year of the end of the course of treatment and those performed without previous chemotherapy. EOR data were calculated as described above.

Perioperative blood loss was approximated using the following formula: $\mathrm{CBL}=\mathrm{RBC}+\mathrm{EBV}-(\mathrm{Hct}$ pre $-\mathrm{Hct}$ 
post), where $\mathrm{CBL}=$ calculated blood loss, $\mathrm{RBC}=$ volume of red blood cells transfused, EBV = estimated blood volume $(80 \mathrm{ml} / \mathrm{kg})$, Hct pre $=$ preoperative hematocrit, and Hct post $=$ postoperative hematocrit. ${ }^{3}$ For clinically relevant comparison, the percentage of blood loss in relation to the patient's estimated total blood volume is reported. Additionally, to assess potential predictors for blood loss, 2 groups were formed, using $25 \%$ of estimated blood volume as a cutoff point (i.e., blood loss $<25 \%$ of estimated blood volume and blood loss $\geq 25 \%$ of estimated blood volume).

\section{Statistical Analysis}

After data collection, the database was deidentified and transferred to IBM SPSS v22 for statistical analysis. Group comparisons were performed using the chi-square/ Fisher exact test for categorical variables and the t-test for comparison of means or the Mann-Whitney U-test for continuous variables. For survival analysis, Kaplan-Meier plots were generated in the R v3.1 statistical environment, using the R packages survival (v2.37) and ggplot2 (v0.9.3.1). An event was defined as death of the patient, and for those who did not die, censoring was performed at the last known follow-up, with right-censoring at 10 years. Time to event or censoring was recorded as years from the date of the first pathologic imaging. Two grouping variables (neoadjuvant chemotherapy and EOR) were compared with regard to survival time by means of the log-rank method. Potential predictors (age, weight, tumor volume resected, and neoadjuvant chemotherapy) for high blood loss ( $\geq 25 \%$ of estimated blood volume) were additionally included in a multivariable logistic regression analysis to identify possible independent predictors. For all statistical tests, significance was assumed at an error probability of less than $5 \%(\mathrm{p}<0.05)$.

Finally, blood loss as percentage of estimated total blood volume is reported in a box-and-whiskers plot, stratified by preoperative use of neoadjuvant chemotherapy, with the 25th, 50th, and 75th percentiles indicated by the box, and the 5th and 95th percentiles indicated by the whiskers.

\section{Results}

From December 1982 to September 2013, 22 consecutive patients were surgically treated for histologically confirmed CPC at the Hospital for Sick Children. Inclusion criteria for this study were met in all cases. Table 1 gives an overview of these cases. Ten patients (45\%) did not receive neoadjuvant chemotherapy, 12 patients $(55 \%)$ were treated with the ICE regimen (ifosfamide $3 \mathrm{~g} / \mathrm{m}^{2}$ on Days 1 and 2, etoposide $150 \mathrm{mg} / \mathrm{m}^{2}$ on Days 1 and 2, and carboplatin $600 \mathrm{mg} / \mathrm{m}^{2}$ on Day 3) in a neoadjuvant fashion. The administration of neoadjuvant chemotherapy delayed resection by a mean of 139 days. A median of 4 neoadjuvant cycles were administered (range 2-5 cycles). In the group of patients who received neoadjuvant chemotherapy, 1 patient underwent gross-total resection and 6 underwent partial resection before chemotherapy, and 5 patients underwent diagnostic biopsy only.

Ten (46\%) of the 22 patients were female. The patients' mean age at diagnosis was 33 months. Ten patients (46\%) were alive at last follow-up and $9(41 \%)$ had died (mean duration of survival after diagnosis for these 9 patients: 20 months). Information on the survival status of 3 patients $(14 \%)$ could not be obtained. Four patients (18\%) had evidence of disseminated disease at presentation, with the reservation that imaging of the whole neuraxis as routine staging has been performed only since the MRI era.

In group comparison, the patients treated with neoadjuvant chemotherapy had a longer mean time to death (38 vs 6 months, $\mathrm{p}=0.01$ ) and a higher rate of gross-total resections $(83 \%$ vs $30 \%, \mathrm{p}=0.03)$. No significant differences could be found between the groups of patients with and without neoadjuvant chemotherapy with respect to the variables sex, age, survival status at follow-up, follow-up time, tumor volume at diagnosis, and administration of radiotherapy.

The results of Kaplan-Meier survival analysis stratified by the parameters "neoadjuvant chemotherapy" and "extent of resection" are reported in Figs. 1 and 2, respectively. Patients treated with neoadjuvant chemotherapy appear to have an improved survival (log-rank $p$ value $=0.03$ ), as do patients with a near-total or gross-total resection (logrank $\mathrm{p}$ value $=0.03$ ).

The 22 patients included in this study underwent a total number of 37 surgeries for tumor resection. Table 2 summarizes these interventions stratified according to the use of neoadjuvant chemotherapy. In comparing resections performed with and without previous chemotherapy, a higher percentage of procedures performed after neoadjuvant chemotherapy resulted gross-total resection $(72 \%$ vs $26 \%, p=0.02)$ and less residual tumor was left $(94 \%$ vs $74 \%$ tumor volume resected, $\mathrm{p}=0.02$; mean residual tumor volumes $0.6 \mathrm{~cm}^{3}$ vs $22.8 \mathrm{~cm}^{3}, \mathrm{p}=0.01$ ). Most prominently, perioperative blood loss was significantly lower after neoadjuvant chemotherapy (mean $22 \%$ vs $96 \%$ of estimated blood volume lost, $\mathrm{p}<0.001$ ). Age, weight, and estimated blood volume did not differ significantly between the 2 groups.

To assess independent predictors for a high blood loss, the parameters of age, weight, tumor volume resected, and neoadjuvant chemotherapy were compared in patients who lost $<25 \%$ versus those who lost $\geq 25 \%$ of their estimated blood volume during surgery (Table 3 ). In a multivariable logistic regression analysis including all 4 variables (age, weight, tumor volume resected, and neoadjuvant chemotherapy), the only independent factor influencing blood loss was the administration of neoadjuvant chemotherapy $(\mathrm{p}=0.03$, OR $10.1,95 \%$ CI $1.2-82.8)$. This association is visualized in Fig. 3, where blood loss in the resections performed after neoadjuvant chemotherapy and resections without previous neoadjuvant chemotherapy is reported in a box-and-whiskers plot. In the surgeries after neoadjuvant chemotherapy, not only is the median blood loss less, but it also appears that extreme amounts of blood loss of several total blood volumes did not occur after administration of neoadjuvant chemotherapy.

\section{Discussion}

Choroid plexus carcinomas remain challenging to treat 
TABLE 1: Summary of clinical and demographic characteristics in patients who underwent resection with or without prior neoadjuvant chemotherapy

\begin{tabular}{|c|c|c|c|c|}
\hline Variable & $\begin{array}{l}\text { Total } \\
(n=22)\end{array}$ & $\begin{array}{l}\text { Pts w/o Neoadjuvant } \\
\text { Chemo }(n=10)\end{array}$ & $\begin{array}{l}\text { Pts w/ Neoadjuvant } \\
\text { Chemo }(n=12)\end{array}$ & $\begin{array}{c}p \\
\text { Value* }\end{array}$ \\
\hline Female & $10(45.5 \%)$ & $4(40.0 \%)$ & $6(50.0 \%)$ & 0.69 \\
\hline Age at Dx (mos) & & & & 0.28 \\
\hline Mean & 33 & 42 & 25 & \\
\hline Median & 26 & 41 & 18 & \\
\hline Status at last follow-up & & & & 0.39 \\
\hline Alive & $10(45.5 \%)$ & $3(30.0 \%)$ & $7(58.3 \%)$ & \\
\hline Deceased & $9(40.9 \%)$ & $5(50.0 \%)$ & $4(33.3 \%)$ & \\
\hline Unknown & $3(13.6 \%)$ & $2(20.0 \%)$ & $1(8.3 \%)$ & \\
\hline Survival time of deceased (mos) & & & & 0.01 \\
\hline Mean & 20 & 6 & 38 & \\
\hline Median & 11 & 6 & 37 & \\
\hline Follow-up time (mos) & & & & 0.15 \\
\hline Mean & 65 & 41 & 84 & \\
\hline Median & 45 & 11 & 55 & \\
\hline Tumor vol at $\mathrm{Dx}\left(\mathrm{cm}^{3}\right)$ & & & & 0.38 \\
\hline Mean & 110 & 92 & 124 & \\
\hline Median & 112 & 104 & 113 & \\
\hline EOR† & & & & 0.03 \\
\hline GTR & $13(59.1 \%)$ & $3(30.0 \%)$ & $10(83.3 \%)$ & \\
\hline NTR (>95\%) & $6(27.3 \%)$ & $4(40.0 \%)$ & $2(16.7 \%)$ & \\
\hline Partial & $3(13.6 \%)$ & $3(30.0 \%)$ & $0(0.0 \%)$ & \\
\hline Preceding surgery in pts w/ neoadjuvant chemo & & & & - \\
\hline GTR & - & - & $1(8.3 \%)$ & \\
\hline NTR (>95\%) & - & - & $0(0.0 \%)$ & \\
\hline Partial & - & - & $6(50.0 \%)$ & \\
\hline Biopsy & - & - & $5(41.7 \%)$ & \\
\hline Time delay due to chemo (days) in pts w/ neoadjuvant chemo & & & & - \\
\hline Mean & - & - & 139 & \\
\hline Median & - & - & 132 & \\
\hline No. of preop ICE cycles in pts w/ neoadjuvant chemo & & & & - \\
\hline Mean & - & - & 3.9 & \\
\hline Median & - & - & 4 & \\
\hline Pts treated w/ XRT after 1st resection & $4(18.2 \%)$ & $3(30.0 \%)$ & $1(8.3 \%)$ & 0.29 \\
\hline Pts treated w/ XRT at any time & $8(36.4 \%)$ & $3(30.0 \%)$ & $5(41.7 \%)$ & 0.68 \\
\hline
\end{tabular}

and have a dismal prognosis in a substantial proportion of patients affected. Based on our data and previous publications, the achievement of a gross-total resection appears to be prognostically favorable. , $^{2,5-10,12,16,17} \mathrm{In}$ fact, all of the long-term survivors in our series underwent a near-total or gross-total resection of their tumor. As CPCs show a close relation to the ventricular system, the anatomical localization is commonly less of a concern when planning resection. Almost invariably, the reason for leaving tumor behind is excessive blood loss, particularly in very young children. In one of the early cases in our series, a patient died of hypovolemic cardiac arrest shortly after surgery.

The administration of neoadjuvant chemotherapy seems to address tumor hypervascularity very effectively. This effect was mentioned by St. Clair et al. in 1991 and confirmed in a series of publications afterward. ${ }^{10,13-15}$ Similarly, the beneficial effect of gross-total resection was published previously. ${ }^{9,10,17}$ Surgeries after neoadjuvant chemotherapy in our patients do show a higher rate of grosstotal resections and smaller blood losses. In addition, the 


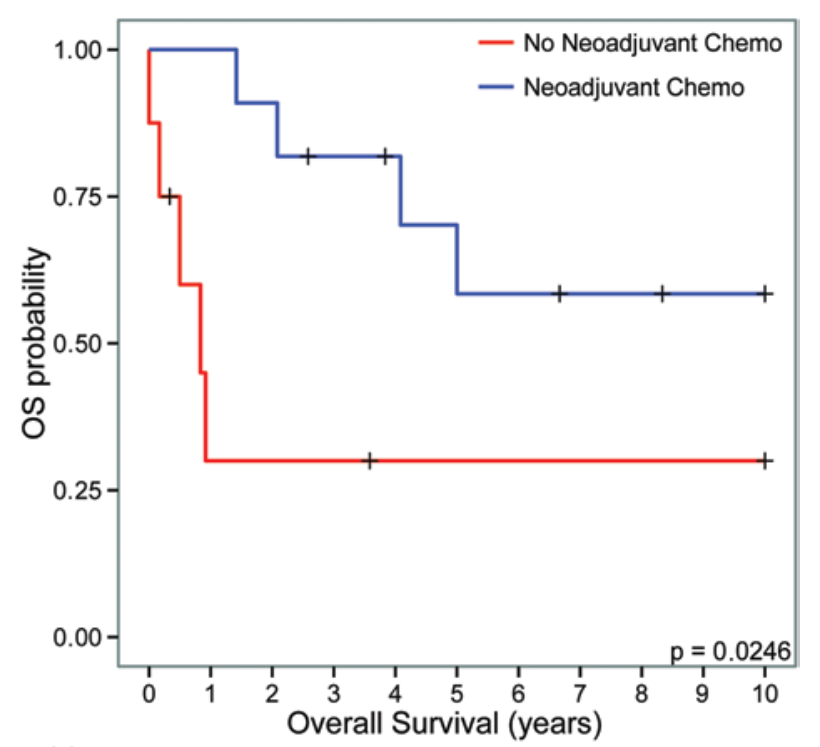

No. at risk

$\begin{array}{lccccccccccc}\text { Chemo } & 11 & 11 & 10 & 8 & 7 & 6 & 5 & 4 & 4 & 3 & 3 \\ \text { No Chemo } & 8 & 2 & 2 & 2 & 1 & 1 & 1 & 1 & 1 & 1 & 1\end{array}$

FIG. 1. Kaplan-Meier survival analysis stratified by the parameter "neoadjuvant chemotherapy." Censored patients (alive at last follow-up) are indicated on the curves. The time axis is right-censored at 10 years. Three patients with missing survival data were excluded. OS = overall survival. Figure is available in color online only.

range of blood loss seems to be limited in the "surgeries with neoadjuvant chemo" group, meaning that extreme losses of over 2 blood volumes did not occur in any surgery performed after chemotherapy in our case series. When potential predictors for high blood loss were included in a logistic regression analysis, only neoadjuvant chemotherapy seemed to independently influence blood loss. This effect is best explained by the histological changes of tumor tissue after chemotherapy, leading to less vascular tissue, possibly a better brain-tumor border, and a blood supply that might be easier to control surgically.

Even when the patient's clinical condition allows for delayed resection, the decision to perform only a diagnostic biopsy obviously has to be made during surgery. At our institution, the preoperative neuroradiologist's opinion combined with the level of certainty uttered by the neuropathologist (based on several frozen sections) ultimately led to the surgeon's decision. Definitive histology was always congruent to the diagnosis obtained from the quick section. However, getting a different definitive histological diagnosis after performing a biopsy only (i.e., plexus papilloma) is certainly a matter of concern. Alternatively, the surgeon might decide to proceed with the resection when feeling comfortable with the bleeding situationeven when a CPC is reported in the frozen section. In light of these considerations, the recommendation for individual surgical planning is to prepare for a tumor resection even when there is a high level of suspicion for a CPC. In these vascular tumors, needle or endoscopic biopsies in our opinion offer only insufficient options for hemostasis when intratumoral bleeding occurs.

Neoadjuvant chemotherapy does have clinical limita-

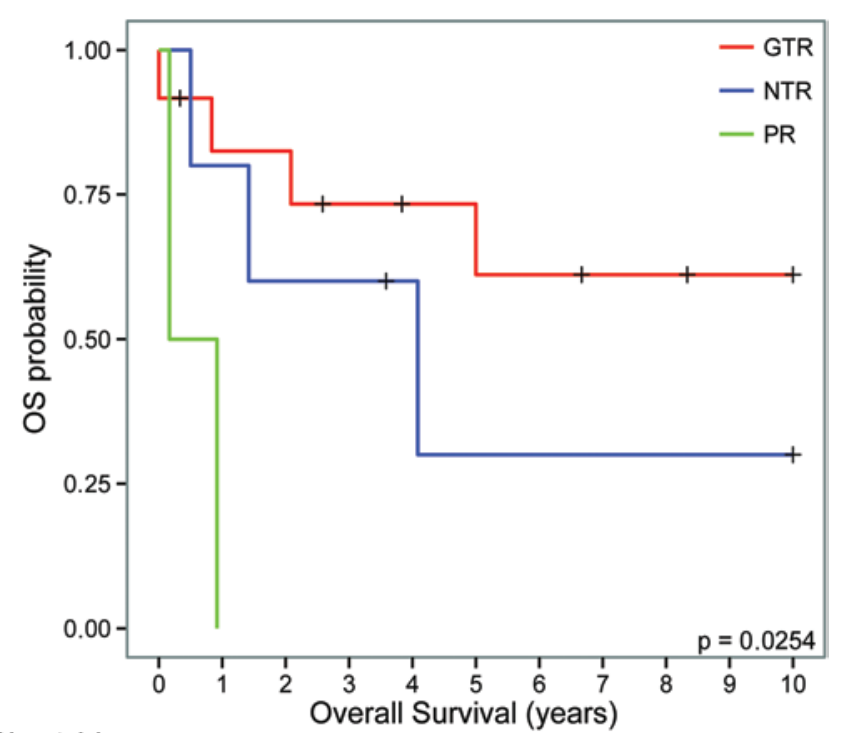

No. at risk

$\begin{array}{lrllllllllll}\text { PR } & 2 & 0 & 0 & 0 & 0 & 0 & 0 & 0 & 0 & 0 & 0 \\ \text { NTR } & 5 & 4 & 3 & 3 & 2 & 1 & 1 & 1 & 1 & 1 & 1 \\ \text { GTR } & 12 & 9 & 9 & 7 & 6 & 6 & 5 & 4 & 4 & 3 & 3\end{array}$

FIG. 2. Kaplan-Meier survival analysis stratified by the parameter "extent of resection." Censored patients (alive at last follow-up) are indicated on the curves. The time axis is right-censored at 10 years. Three patients with missing survival data were excluded. GTR $=$ gross-total resection; NTR = near-total resection; PR = partial resection. Figure is available in color online only.

tions. CPCs often present with CSF circulation impairment, and a presentation in extremis with decompensated obstructive hydrocephalus is not uncommon. The mean delay to definitive surgery due to the administration of neoadjuvant chemotherapy with the ICE regimen was 139 days in our series, time that might not be available in some cases. CSF diversion surgery or tumor debulking may be inevitable as an emergency treatment. Therefore it might be argued that patients in whom clinical status allows for biopsy, chemotherapy, and delayed resection do have a much better baseline than those who require emergent intervention and that these 2 groups are not comparable. We tried to overcome this possible bias with the definition of the "patients with neoadjuvant chemo" group. The only criterion for assignment to this group was resection following chemotherapy within 1 year. This group does include patients with a low neurological baseline who had to undergo an intervention for tumor debulking and CSF diversion dictated by clinical necessity and who underwent a second resection after administration of chemotherapy. It appears that these patients do not fare worse in terms of overall survival than those whose cases could be "ideally" managed by biopsy followed by chemotherapy followed by resection.

The second argument against performing a diagnostic biopsy as a first intervention is in fact again the high vascularity of CPC and the often large size of these tumors at presentation. Of the 5 patients who initially underwent only biopsy procedures rather than resection, 2 suffered severe complications. One developed malignant brain edema requiring decompressive craniectomy, and one suf- 
TABLE 2. Resections-overview*

\begin{tabular}{|c|c|c|c|c|}
\hline Variable & Total $(n=37)$ & $\begin{array}{l}\text { Resection w/o Neoadjuvant } \\
\text { Chemo }(n=19)\end{array}$ & $\begin{array}{l}\text { Resection After Neoadjuvant } \\
\text { Chemo }(n=18)\end{array}$ & $\mathrm{p}$ Value \\
\hline Pt age at resection (mos) & & & & 0.64 \\
\hline Mean & 39 & 43 & 36 & \\
\hline Median & 28 & 28 & 28 & \\
\hline EOR & & & & 0.02 \\
\hline GTR & $18(48.6)$ & $5(26.3)$ & $13(72.2)$ & \\
\hline NTR (>95\%) & $6(16.2)$ & $5(26.3)$ & $1(5.6)$ & \\
\hline Partial & $13(35.1)$ & $9(47.4)$ & $4(22.2)$ & \\
\hline$\%$ of tumor vol resected (mean) & 83 & 74 & 94 & 0.02 \\
\hline Residual tumor vol in $\mathrm{cm}^{3}$ (mean) & 12.0 & 22.8 & 0.6 & 0.01 \\
\hline Pt weight at op (kg) & & & & 0.74 \\
\hline Mean & 16 & 15 & 16 & \\
\hline Median & 12 & 12 & 13 & \\
\hline $\mathrm{EBV}(\mathrm{ml})$ & & & & 0.74 \\
\hline Mean & 1252 & 1202 & 1305 & \\
\hline Median & 984 & 984 & 1004 & \\
\hline Blood loss, $\%$ of EBV (mean) & 60 & 96 & 22 & $<0.01$ \\
\hline
\end{tabular}

fered an intratumoral hemorrhage needing emergent surgical evacuation. Therefore, the benefit for the patient with neoadjuvant chemotherapy in allowing for the best possible resection with minimized blood loss has to be carefully balanced against the risk of not performing volume reduction of a cerebral mass lesion. This decision should be made on a case-by-case basis, depending on the patient's clinical status, the size of the tumor, and the degree of hydrocephalus. In our series, the patients who tolerated delayed surgery best were-not surprisingly-those with supratentorial tumors of small or approximately median size and only moderate hydrocephalus. Keeping in mind these limitations, the substantial reduction of intraoperative blood loss and the increased likelihood of a gross-total resection provides ample evidence to support widespread adoption of the neoadjuvant regimen in patients with CPC.

One limitation of this manuscript is the nonrandom assignment of the patients into treatment groups. The decision to administer neoadjuvant chemotherapy was influenced by many factors. In the early cases in this series, this option was not considered at all. This means that the benefit of a resection after chemotherapy only became evident at the second attempt at resection, when a first attempt at gross-total resection had failed because of high blood loss

TABLE 3. Resections-potential predictors for high blood loss*

\begin{tabular}{|c|c|c|c|c|}
\hline Variable & $\begin{array}{c}\text { Blood Loss } \geq 25 \% \text { of EBV } \\
\qquad(n=19)\end{array}$ & $\begin{array}{l}\text { Blood Loss }<25 \% \text { of EBV } \\
\qquad(n=18)\end{array}$ & p Value & p Value (logistic regression) \\
\hline Age at resection (mos) & & & 0.07 & 0.63 \\
\hline Mean & 26 & 54 & & \\
\hline Median & 19 & 37 & & \\
\hline Weight at resection $(\mathrm{kg})$ & & & 0.02 & 0.26 \\
\hline Mean & 11 & 20 & & \\
\hline Median & 10 & 14 & & \\
\hline Tumor vol resected $\left(\mathrm{cm}^{3}\right)$ & & & 0.06 & 0.10 \\
\hline Mean & 90 & 43 & & \\
\hline Median & 70 & 7 & & \\
\hline Neoadjuvant chemo & & & $<0.01$ & 0.03 \\
\hline Mean & 4 & 14 & & \\
\hline Median & 21.1 & 77.8 & & \\
\hline
\end{tabular}

\footnotetext{
* Bold type indicates statistical significance.
} 


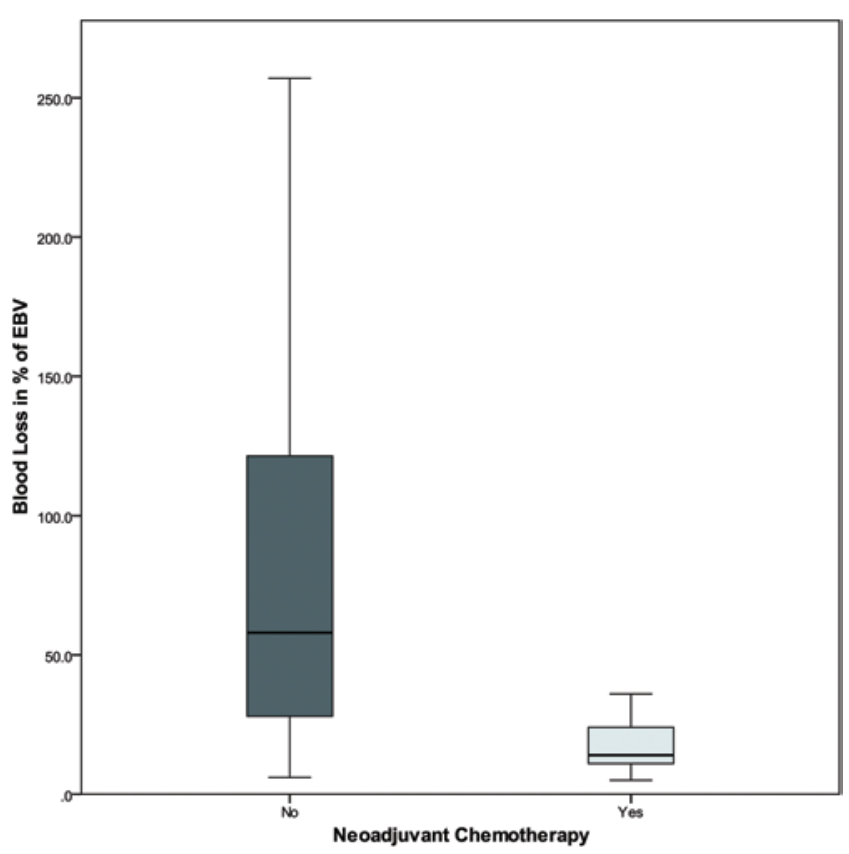

FIG. 3. Box-and-whiskers plots showing blood loss in 37 choroid plexus carcinoma resections stratified by the administration of neoadjuvant chemotherapy. The 25th, 50th, and 75th percentiles are indicated by the boxes and the 5th and 95th by the whiskers. Mann-Whitney U-test, $p<$ 0.001 . EBV $=$ estimated blood volume. Figure is available in color online only.

or when the patient showed recurrence after adjuvant chemotherapy. Intentional diagnostic biopsies with the plan to administer neoadjuvant chemotherapy were performed at our institution beginning in 1999. Since then, when a diagnostic biopsy was the intention, clinical necessity sometimes forced the surgeon to alter the plan.

In addition, the observational period spans 3 decades. Many other influencing factors have improved dramatically over this long time (neuroimaging, surgical technique, neuroanaesthesiology). Therefore, the best statement that can be made is that in this heterogeneous retrospective patient series, surgery following chemotherapy was associated with greatly decreased intraoperative blood loss and increased overall survival.

\section{Conclusions}

In a retrospective review of 22 pediatric patients with CPC, the administration of neoadjuvant ICE chemotherapy was associated with dramatic reduction of intraoperative blood loss (from $96 \%$ of estimated total blood volume to $22 \%$ ) in a total of 37 tumor resection procedures. This effect is - among others-likely to explain the much higher incidence of gross-total resections in the neoadjuvant chemotherapy group (83\% versus $30 \%$ ). Patients who did receive a gross-total resection showed a longer overall survival time.

Based on these observations, the administration of neoadjuvant chemotherapy after diagnostic biopsy is recommended in patients who do not need emergent tumor volume reduction. When debulking is inevitable at presentation, a second resection after chemotherapy is likely to result in a gross-total resection while minimizing blood loss and provides the best chance for long-term tumor-free survival.

\section{References}

1. Allen J, Wisoff J, Helson L, Pearce J, Arenson E: Choroid plexus carcinoma-responses to chemotherapy alone in newly diagnosed young children. J Neurooncol 12:69-74, 1992

2. Berger C, Thiesse P, Lellouch-Tubiana A, Kalifa C, PierreKahn A, Bouffet E: Choroid plexus carcinomas in childhood: clinical features and prognostic factors. Neurosurgery 42:470-475, 1998

3. Brecher ME, Monk T, Goodnough LT: A standardized method for calculating blood loss. Transfusion 37:1070-1074, 1997

4. Dohrmann GJ, Collias JC: Choroid plexus carcinoma. J Neurosurg 43:225-232, 1975

5. Due-Tønnessen B, Helseth E, Skullerud K, Lundar T: Choroid plexus tumors in children and young adults: report of 16 consecutive cases. Childs Nerv Syst 17:252-256, 2001

6. Duffner PK, Kun LE, Burger PC, Horowitz ME, Cohen ME, Sanford RA, et al: Postoperative chemotherapy and delayed radiation in infants and very young children with choroid plexus carcinomas. Pediatr Neurosurg 22:189-196, 1995

7. Fitzpatrick LK, Aronson LJ, Cohen KJ: Is there a requirement for adjuvant therapy for choroid plexus carcinoma that has been completely resected? J Neurooncol 57:123-126, 2002

8. Koh EJ, Wang KC, Phi JH, Lee JY, Choi JW, Park SH, et al: Clinical outcome of pediatric choroid plexus tumors: retrospective analysis from a single institute. Childs Nerv Syst 30:217-225, 2014

9. Lafay-Cousin L, Keene D, Carret AS, Fryer C, Brossard J, Crooks B, et al: Choroid plexus tumors in children less than 36 months: the Canadian Pediatric Brain Tumor Consortium (CPBTC) experience. Childs Nerv Syst 27:259-264, 2011

10. Lafay-Cousin L, Mabbott DJ, Halliday W, Taylor MD, Tabori U, Kamaly-Asl ID, et al: Use of ifosfamide, carboplatin, and etoposide chemotherapy in choroid plexus carcinoma. J Neurosurg Pediatr 5:615-621, 2010

11. Mosleh O, Tabori U, Bartels U, Huang A, Schechter T, Bouffet E: Successful treatment of a recurrent choroid plexus carcinoma with surgery followed by high-dose chemotherapy and stem cell rescue. Pediatr Hematol Oncol 30:386-391, 2013

12. Packer RJ, Perilongo G, Johnson D, Sutton LN, Vezina G, Zimmerman RA, et al: Choroid plexus carcinoma of childhood. Cancer 69:580-585, 1992

13. Razzaq AA, Cohen AR: Neoadjuvant chemotherapy for hypervascular malignant brain tumors of childhood. Pediatr Neurosurg 27:296-303, 1997

14. Souweidane MM, Johnson JH Jr, Lis E: Volumetric reduction of a choroid plexus carcinoma using preoperative chemotherapy. J Neurooncol 43:167-171, 1999

15. St Clair SK, Humphreys RP, Pillay PK, Hoffman HJ, Blaser SI, Becker LE: Current management of choroid plexus carcinoma in children. Pediatr Neurosurg 17:225-233, 1991-1992

16. Sun MZ, Ivan ME, Clark AJ, Oh MC, Delance AR, Oh T, et al: Gross total resection improves overall survival in children with choroid plexus carcinoma. J Neurooncol 116:179-185, 2014

17. Sun MZ, Oh MC, Ivan ME, Kaur G, Safaee M, Kim JM, et 
al: Current management of choroid plexus carcinomas. Neurosurg Rev 37:179-192, 2014

18. Wolff JE, Sajedi M, Coppes MJ, Anderson RA, Egeler RM: Radiation therapy and survival in choroid plexus carcinoma. Lancet 353:2126, 1999

\section{Author Contributions}

Conception and design: Schneider, Kamaly-Asl, Ramaswamy, Lafay-Cousin, Kulkarni, Rutka, Tabori, Hawkins, Bouffet, Taylor. Acquisition of data: Schneider, Kamaly-Asl, Ramaswamy, Lafay-Cousin, Tabori, Bouffet . Analysis and interpretation of data: Schneider, Kamaly-Asl, Ramaswamy, Lafay-Cousin, Kulkarni, Hawkins, Taylor. Drafting the article: Schneider, Kamaly-Asl, Ramaswamy, Kulkarni, Rutka, Remke, Coluccia, Tabori, Bouffet, Taylor. Critically revising the article: Schneider, Kamaly-Asl, Ramaswamy, Kulkarni, Rutka, Remke,
Coluccia, Tabori, Bouffet, Taylor. Reviewed submitted version of manuscript: Schneider, Ramaswamy, Kulkarni, Remke, Coluccia, Taylor. Approved the final version of the manuscript on behalf of all authors: Schneider. Statistical analysis: Schneider, KamalyAsl, Ramaswamy, Lafay-Cousin, Kulkarni. Administrative/ technical/material support: Schneider, Kamaly-Asl, Ramaswamy, Lafay-Cousin, Kulkarni, Tabori, Hawkins, Bouffet, Taylor. Study supervision: Bouffet, Taylor.

\section{Supplemental Information}

Previous Presentation

Portions of this work were presented at the International Society for Pediatric Neurosurgery Meeting, Vancouver, September 2005.

\section{Correspondence}

Christian Schneider, Division of Neurosurgery, The Hospital for Sick Children, 555 University Ave., Toronto, ON M5G 1X8,

Canada. email: christian_schneider@gmx.ch. 